\title{
Child and parental executive functioning in type 1 diabetes: Their unique and interactive role toward treatment adherence and glycemic control
}

\author{
Eveline R Goethals ${ }^{1,2}$ @ | Maartje de Wit ${ }^{3}$ | Nady Van Broeck ${ }^{1}$ | Jurgen Lemiere ${ }^{1,2}$ | \\ Dagmar Van Liefferinge $^{1}$ | Susanne Böhler ${ }^{4}$ | Marian De wulf ${ }^{5}$ | Elke Dello ${ }^{6}$ | \\ Jolien Laridaen ${ }^{7}$ | Lynn Van Hecke ${ }^{8}$ | Shana Van Impe ${ }^{9}$ | Kristina Casteels ${ }^{2,10}$ | \\ Koen Luyckx ${ }^{1}$
}

${ }^{1}$ Faculty of Psychology and Educational Sciences, University of Leuven, Leuven, Belgium

${ }^{2}$ Department of Pediatrics, University Hospitals Leuven, Leuven, Belgium

${ }^{3}$ Department of Medical Psychology, VU University Medical Center, Amsterdam, The Netherlands

${ }^{4}$ Department of Pediatrics, University Hospital Brussels, Brussels, Belgium

${ }^{5}$ Department of Pediatrics, Antwerp University Hospital, Edegem, Belgium

${ }^{6}$ Department of Pediatrics, Hospital Maas \& Kempen, Bree, Belgium

${ }^{7}$ Department of Pediatrics, University Hospital Ghent, Ghent, Belgium

${ }^{8}$ Department of Pediatrics, Delta Hospital Roeselare, Roeselare, Belgium

${ }^{9}$ Department of Pediatrics, Queen Paola Children's Hospital ZNA, Antwerp, Belgium

${ }^{10}$ Department of Development and Regeneration, University of Leuven, Leuven, Belgium

Correspondence

Eveline R Goethals, Department of Pediatrics, University Hospitals Leuven, Herestraat 49, 3000 Leuven, Belgium.

Email: Eveline.Goethals@uzleuven.be

Funding information

Clinical Research Fund (University Hospitals Leuven); VDKA (Vlaamse Diabetescentra voor Kinderen en Adolescenten).
Objective: Managing type 1 diabetes (T1D) requires the ability to make complex and critical decisions regarding treatment, to execute complex tasks accurately, and to make adjustments when problems arise. This requires effective neuropsychological competences of patients and their families, especially in the domain of executive functioning (EF): the ability to self-monitor, plan, solve problems, and set priorities. Previous research focused mainly on child EF, neglecting the impact of parental EF. This study included both mothers and fathers and examined associations between child and parental EF and treatment adherence to T1D in a broad age range of patients.

Methods: Parents of 270 patients (6-18 years) with T1D (mean age 12.7 years; $52.6 \%$ female) were included. Mothers $(N=232)$ and fathers $(N=168)$ completed questionnaires on child and parental EF and on treatment adherence. Analyses examined the associations linking child and parental EF to treatment adherence and glycemic control (and potential moderation effects in these associations) using hierarchical linear regression.

Results: Child EF problems were negatively associated with treatment adherence. As an indication of moderation, this effect was stronger in older children. Better treatment adherence and glycemic control were reported when both child and parent showed less EF problems. Effects were more pronounced in mothers than in fathers.

Conclusions: This study demonstrated a significant interplay between child and parental EF in the association with treatment adherence and glycemic control. Researchers and clinicians should remain attentive toward the role of neuropsychological concepts such as EF. Implementation in clinical practice seems meaningful.

\section{KEYWORDS}

executive functioning, treatment adherence

\section{1 | INTRODUCTION}

Treatment of type 1 diabetes (T1D) imposes many behavioral demands on young patients and their families. ${ }^{1}$ Treatment non- adherence in children and adolescents is high and is associated with short- and long-term adverse health outcomes. ${ }^{2-4}$ Technological improvements in insulin pumps and continuous glucose monitoring make treatment more individually tailored and help in managing 
T1D. ${ }^{2,5}$ However, they also increase the complexity of treatment. Children's and families' disease knowledge and skills for managing the disease are critical for treatment adherence. Active knowledge requires an accurate understanding and mastering of the tasks that are involved in treatment management. ${ }^{6}$ Patients need to make complex and critical decisions regarding their treatment, execute complex tasks accurately, and make adjustments when problems arise. ${ }^{6-8}$ These specific cognitive demands are captured by the multifaceted construct of executive functioning (EF). EF involves various high-level self-regulatory cognitive processes related to frontal cortical functions that control and organize lower-order mental processes. ${ }^{7,9}$ It includes the facilitation of new ways of behaving and optimization of one's approach to unfamiliar circumstances, the ability to self-monitor, focus attention, remember instructions, plan, and use working memory. ${ }^{7,10,11}$ Given that higher cortical maturation continues into adulthood, ${ }^{10,12}$ the management of T1D might at some point demand more of children and adolescents than they are cognitively capable of. Although managing T1D is considered difficult at any age, adolescents especially, are at increased risk for experiencing difficulty adhering to the treatment regimen and for suboptimal glycemic control. $^{7,13,14}$ Research indicates that although cortical maturation increases in adolescence, EF competencies may decrease due to conflicting developmental challenges (eg, more peer-oriented behavior ${ }^{15}$ ). Moreover, whereas young children depend completely on their parents, this dependence increasingly shifts toward shared responsibility and decision-making in adolescence. ${ }^{16,17}$

Research on the role of EF in treatment adherence to T1D is mounting but remains relatively scarce. Neuropsychological studies in children and adolescents with T1D have established evidence for weaker cognition compared to healthy controls and even for structural changes in the brain. ${ }^{18-20}$ Hence, decreased treatment adherence may be a reflection of impairments in neuropsychological functioning. ${ }^{8}$ In terms of EF more specifically, there is research suggesting a bidirectional negative association between EF of children and adolescents, treatment adherence, and glycemic control. ${ }^{7,21,22}$ However, results across studies are inconsistent and effect sizes vary from small to medium. Moreover, parental EF has not been taken into account and most research on child EF is based upon mother's and not father's report, although there is extensive literature emphasizing the importance of developmentally appropriate maternal and paternal involvement in treatment adherence to T1D. ${ }^{23,24}$

\section{1 | This study}

As previous studies on EF in children and adolescents with T1D included mainly mother reports, ${ }^{25}$ the current multi-informant study included both parents. Further, previous studies mainly included a small age range. ${ }^{7}$ To take into account a developmental perspective, this study included children and adolescents (6-18 years old), allowing for examining age as a moderating factor. Finally, as parents play a significant role in the treatment of their children's T1D (and especially so in younger children), this study will investigate parental EF as well.

Three objectives guided this study. (1) To examine the main effects of child and parental EF problems on treatment adherence and glycemic control (hemoglobin $\mathrm{A} 1 \mathrm{c}[\mathrm{HbA} 1 \mathrm{c}])$. We hypothesized that child and parental EF problems would be associated with poorer treatment adherence and HbA1c. (2) To examine 3 possible 2-way interaction effects, that is, whether parental EF problems and child age would act as moderators in the link between child EF problems and treatment adherence/glycemic control, and whether child age would act as moderator in the link between parental EF problems and treatment adherence/glycemic control. First, we hypothesized that the negative impact of child EF problems on treatment adherence may be enhanced or compensated when parents score either low or high on EF problems. Second, we hypothesized that the relationship between child EF problems and treatment adherence might be stronger in older children, whereas the effect of parental EF problems would be stronger in the younger age group. (3) To examine the 3-way interaction of child EF problems, parental EF problems, and child age. We hypothesized that in the younger age group, the impact of parental EF problems on the link between child EF problems, treatment adherence, and glycemic control, would be more pronounced than in the older age group.

\section{2 | METHODS}

\section{1 | Participants and procedure}

Mothers and fathers of children and adolescents with T1D meeting the following criteria: (1) having a diagnosis of T1D for at least 6 months, (2) aged 6-18 years, and (3) Dutch-speaking, were recruited from 7 Belgian hospitals. Patients with other severe somatic diagnoses (eg, cystic fibrosis), cognitive disabilities, and/or other psychiatric diagnoses known to be related to problems in overall EF (attention deficit hyperactivity disorder [ADHD] and autism ${ }^{26}$ were excluded. Parents of patients meeting the inclusion criteria were contacted through e-mail with a link to the online questionnaires using Limesurvey. All participants signed an online informed consent form. The study was approved by central and local Institutional Review Boards of all participating centers and in accordance with the Declaration of Helsinki.

\section{2 | Measures}

\subsection{1 | Demographic and diabetes characteristics}

Information on age, sex, ethnicity, educational and professional level, and family situation was completed by parents. Illness duration and glycemic control in terms of $\mathrm{HbA1c}$ value were obtained through chart review by treating physicians for whom participants gave informed consent. HbA1c-values closest to the date the parents filled out the questionnaires (within 3 months before or after questionnaire completion) were collected. In Belgium, continuity and quality of $\mathrm{HbA} 1 \mathrm{c}$ results are guaranteed as all clinical sites have similar assays to measure $\mathrm{HbA1c}$. Further, all Belgian laboratories are obliged to participate in an external quality assessment regarding $\mathrm{HbA} 1 \mathrm{c}$ determination.

\subsection{2 | EF problems child}

The Behavior Rating Inventory of Executive Function-Parent Report (BRIEF-P ${ }^{27}$ ) assesses EF and self-regulation skills in children and adolescents aged 5 to 18 years old through parent reports. Questionnaires contain 86 items and can be divided in 8 non-overlapping clinical scales (inhibit, shift, emotional control, initiate, working 
memory, plan/organize, organization of materials, and monitor) from which 2 broader indexes (behavioral regulation and metacognition) and a global executive composite score can be derived. Answers in terms of "never," "sometimes," or "often" indicate problems in the different areas described over the last 6 months. Higher scores indicate worse EF. In this study, total composite scores indicating global EF were used. Reliabilities of the global EF were high in both mother and father report: $\alpha=.97$.

\subsection{3 | EF problems parent}

The Behavior Rating Inventory of Executive Function-Adult (BRIEF$\left.A^{28}\right)$ is a self-report questionnaire assessing EF and self-regulation capacities in adults from 18 to 90 years of age. It consists of 75 items and, similarly to the BRIEF-P, can be divided in 9 clinical scales and 2 broader indexes. Reliabilities of the global executive composite score were high: $\alpha=.95$ and $\alpha=.96$ (mother and father report).

\subsection{4 $\mid$ Treatment adherence}

The Diabetes Self-Management Profile-Self-Report (DSMP-SR ${ }^{29}$ ) is a 24-item measure quantifying self-management behaviors over the past 3 months as reported upon by parents. It was translated in Dutch using the back-translation procedure. Items are rated on 2, 3, or 4 point Likert response scales. Total adherence scores were calculated ranging from 0 to 86 with higher scores indicating better treatment adherence. Reliabilities were adequate: $\alpha=.70$ and $\alpha=.67$ (mother and father report).

\section{3 | Statistical analyses}

Statistical analyses were performed using SPSS v.24. Four sets of hierarchical regression analyses were conducted to examine the contribution of EF problems child, EF problems parent, and age-and their interactions-in the prediction of mother and father reports of treatment adherence and $\mathrm{HbA1c}$.

All independent variables except for gender ( 0 = female; 1 = male) were standardized. ${ }^{30}$ In step 1 , we added gender, age, and illness duration. In step 2, main effects of child and parental EF problems were added. In step 3, 3 2-way interaction terms were added: EF problems child $\times E F$ problems parent, EF problems child $\times$ age child, and EF problems parent $\times$ age child. Finally, in step 4, the 3-way interaction was added: EF problems child $\times$ EF problems parent $\times$ age child. In steps 3 and 4 , we interpreted individual significant interaction terms if the chunk test indicated a significant increase in $R^{2}{ }^{31}$

\section{3 | RESULTS}

\section{1 | Patient characteristics}

Parents of 766 patients meeting the inclusion criteria were contacted through e-mail. Of 270 (35.2\%) patients at least one parent completed the questionnaires: 232 mothers and 168 fathers. Of 116 patients both mother and father completed all the questionnaires. About half of the patients were female $(N=142 ; 52.6 \%)$ and patients were 12.70 years old on average $(\mathrm{SD}=3.27)$. Mean $\mathrm{HbA} 1 \mathrm{c}$ was 7.32 $(\mathrm{SD}=0.98) ;$ median $=7.30([6.7 ; 7.9] ; 56 \mathrm{mmol} / \mathrm{mol})$. Mean illness duration was 5.46 years $(S D=3.61)$. The minority of patients administered insulin by pump therapy (25.4\%). For additional participants' characteristics, see Table 1.

\section{2 | Correlation analyses}

Table 2 displays Pearson correlations among the study variables. A significant negative correlation was found between child as well as parental EF problems and treatment adherence within respondents (ie, same parent reporting on both variables). A consistent significant positive correlation was also found between mother and father reports of EF problems child, EF problems parent, and treatment adherence. Better treatment adherence was correlated with lower $\mathrm{HbA1c}$, younger age, and longer illness duration.

\section{3 | Regression analyses}

Standardized betas and $R^{2}$-values for mother and father reports are presented in Tables 3 and 4. Figure 1 shows graphical depictions of all significant 2-way and 3-way interactions.

\subsection{1 | Mother reports}

In step 1, gender was not significantly related to treatment adherence or $\mathrm{HbA1c}$, indicating that there were no differences between girls and boys with regard to treatment adherence and $\mathrm{HbA1C}$. Age and illness duration were negatively related to treatment adherence and positively to HbA1c. In step 2, EF problems child was significantly negatively related to mother reports of treatment adherence and positively to $\mathrm{HbA1c}$. On the contrary, maternal EF problems were not related to treatment adherence and HbA1c. In step 3, 3 2-way interactions were examined. First, the 2-way interaction between EF problems child and EF problems parent reached significance for mother and father reports of treatment adherence and marginal significance for $\operatorname{HbA1C}(P<.10)$. As displayed in Figure $1 \mathrm{~A}, \mathrm{~B}$, the highest level of treatment adherence was reached when the child as well as the mother experienced less problems in EF. Second, the 2-way interaction between child EF problems and age also reached significance for (mother but not father report of) treatment adherence and HbA1c. Hence, as displayed in Figure 1C,D, EF problems child were related

TABLE 1 Participants' characteristics

\begin{tabular}{|llll} 
& $\begin{array}{l}\text { Patients } \\
(n=270)\end{array}$ & $\begin{array}{l}\text { Mothers } \\
(n=232)\end{array}$ & $\begin{array}{l}\text { Fathers } \\
(n=168)\end{array}$ \\
\hline Age & $12.70(3.27)$ & $43.12(4.74)$ & $45.01(5.49)$ \\
\hline HbA1c (\%) & $7.32(0.98)$ & & \\
\hline Illness duration & $5.46(3.61)$ & & \\
\hline Pump therapy & 25.4 & & 84.4 \\
\hline Intact family & 88.5 & 83.2 & 89.29 \\
\hline Belgian nationality & 95.6 & 93.53 & 63.58 \\
\hline Education & & & 35.81 \\
\hline \multicolumn{1}{l}{ University or college } & & 66.67 & 0.61 \\
\hline \multicolumn{1}{c}{ Secondary education } & & 32.9 & 95.67 \\
\hline \multicolumn{1}{l}{ Primary education } & & 0.43 & \\
\hline Working & & 88.31 & \\
\hline
\end{tabular}

Abbreviation: HbA1c, hemoglobin A1c.

Data represent mean (SD) or \%. 
TABLE 2 Intercorrelations, means, and SD for scores on diabetes characteristics, the BRIEF (child and parent), and DSMP-SR

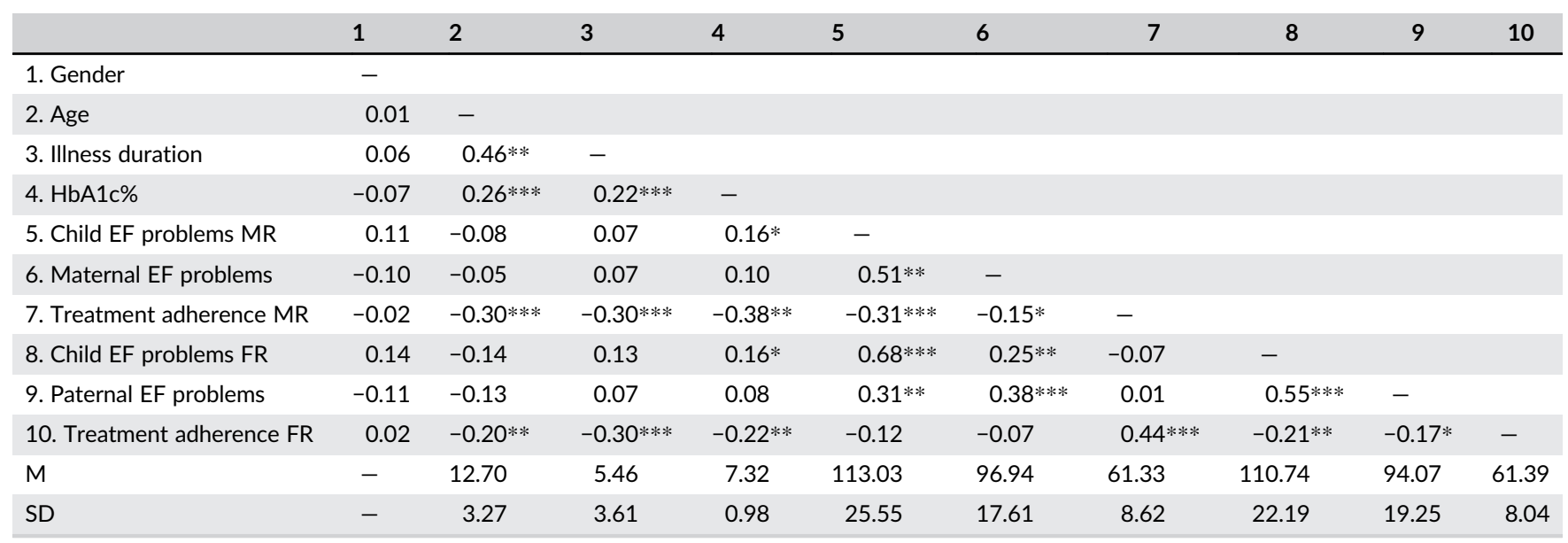

Abbreviations: BRIEF, Behavior Rating Inventory of Executive Function; DSMP-SR, Diabetes Self-Management Profile-Self Report; EF, executive functioning; FR, father report; HbA1c, hemoglobin A1c; MR, mother report.

$* P<.05 ; * * P<.01 ; * * * P<.001$.

to treatment adherence and $\mathrm{HbA} 1 \mathrm{c}$ especially in older children: the worst treatment adherence was reached in older children experiencing problems in EF. Third, the 2-way interaction between EF problems parent and age did not reach significance (unless for a marginally significant interaction in the link with $\mathrm{HbA1c}, P<.10$ ). In step 4, the 3-way interaction effect EF problems child $\times$ EF problems parent $\times$ age was significant for $\mathrm{HbA} 1 \mathrm{c}$ and marginally significant $(P<.10)$ for father reports of treatment adherence. Hence, as displayed in Figure 1D, the highest levels of $\mathrm{HbA} 1 \mathrm{c}$ were attained in older children manifesting more EF problems, and mothers manifesting less problems in the EF domain.

TABLE 3 Standardized beta coefficients from the hierarchical regression analyses for mother reports

\begin{tabular}{|c|c|c|c|}
\hline & $\begin{array}{c}\text { Treatment } \\
\text { adherence MR }\end{array}$ & $\begin{array}{c}\text { Treatment } \\
\text { adherence FR }\end{array}$ & HbA1c \\
\hline Step 1: controls $\left(R^{2}\right)$ & $0.12 * * *$ & 0.06 & $0.09 * * *$ \\
\hline $\begin{array}{l}\text { Gender }(0=\text { female; } \\
1=\text { male })\end{array}$ & 0.00 & 0.11 &.-07 \\
\hline Age & $-0.22 * * *$ & -0.04 & $0.17^{* *}$ \\
\hline Illness duration & $-0.19 * *$ & $-0.20^{+}$ & $0.18 * *$ \\
\hline $\begin{array}{l}\text { Step 2: main effects } \\
\text { (change } R^{2} \text { ) }\end{array}$ & $0.11 * * * *$ & 0.02 & $0.03 * *$ \\
\hline EF problems child & $-0.35 * * *$ & -0.14 & $0.16 * *$ \\
\hline EF problems parent & 0.04 & 0.01 & 0.01 \\
\hline $\begin{array}{l}\text { Step 3: 2-way interactions } \\
\text { (change } R^{2} \text { ) }\end{array}$ & $0.09 * * *$ & $0.08 *$ & $0.06 * * *$ \\
\hline $\begin{array}{l}\text { EF problems child } \times \\
\text { EF problems parent }\end{array}$ & $0.28 * * *$ & $0.27 *$ & $-0.14^{t}$ \\
\hline EF problems child $\times$ age & $-0.17 * *$ & 0.11 & $0.23 * * *$ \\
\hline EF problems parent $\times$ age & 0.05 & -0.15 & $-0.13^{+}$ \\
\hline $\begin{array}{l}\text { Step 4: 3-way } \\
\quad \text { interactions (change } R^{2} \text { ) }\end{array}$ & 0.00 & $0.03^{+}$ & $0.02 * *$ \\
\hline $\begin{array}{l}\text { EF problems child } \times \\
\text { EF problems } \\
\text { parent } \times \text { age }\end{array}$ & 0.03 & $0.25^{+}$ & $-0.17 * *$ \\
\hline
\end{tabular}

Abbreviations: $\mathrm{EF}$, executive functioning; $\mathrm{FR}$, father report; $\mathrm{HbA} 1 \mathrm{c}$, hemoglobin A1c; MR, mother report.

$t_{P}<.10 ; * P<.05 ; * * P<.01 ; * * * P<.001$.

\subsection{2 | Father reports}

In step 1, gender and age were not significantly related to treatment adherence or $\mathrm{HbA1c}$ whereas illness duration was negatively related to treatment adherence and positively to HbA1c. In step 2, EF problems child were not significantly related to treatment adherence and marginally significantly to $\mathrm{HbA} 1 \mathrm{c}(P<.10)$. The main effect of paternal EF problems also did not explain a significant additional proportion of variance in treatment adherence and HbA1c. In step 3, the 2-way interaction between EF problems child and paternal EF problems reached marginal significance $(P<.10)$ with father's report of treatment adherence as outcome variable. This interaction effect was not

TABLE 4 Standardized beta coefficients from the hierarchical regression analyses for father reports

\begin{tabular}{|c|c|c|c|}
\hline & $\begin{array}{c}\text { Treatment } \\
\text { adherence FR }\end{array}$ & $\begin{array}{c}\text { Treatment } \\
\text { adherence MR }\end{array}$ & HbA1c \\
\hline Step 1: controls $\left(R^{2}\right)$ & $0.10 * * *$ & $0.15^{* * *}$ & $0.11 * * *$ \\
\hline $\begin{array}{l}\text { Gender }(0=\text { female; } \\
1=\text { male })\end{array}$ & 0.08 & 0.05 & -0.05 \\
\hline Age & -0.09 & $0.23 *$ & 0.13 \\
\hline Illness duration & $-0.26 * * *$ & $-0.22 *$ & $0.25 * *$ \\
\hline $\begin{array}{l}\text { Step 2: main effects } \\
\text { (change } R^{2} \text { ) }\end{array}$ & $0.04 * *$ & 0.00 & 0.02 \\
\hline EF problems child & -0.12 & -0.08 & $0.17^{+}$ \\
\hline EF problems parent & -0.12 & 0.05 & -0.03 \\
\hline $\begin{array}{l}\text { Step 3: 2-way interactions } \\
\text { (change } R^{2} \text { ) }\end{array}$ & 0.03 & 0.02 & 0.03 \\
\hline $\begin{array}{l}\text { EF problems child } \times \\
\text { EF problems parent }\end{array}$ & $0.17^{+}$ & 0.14 & -0.08 \\
\hline EF problems child $\times$ age & 0.03 & 0.16 & 0.12 \\
\hline EF problems parent $\times$ age & -0.08 & -0.10 & 0.00 \\
\hline $\begin{array}{l}\text { Step 4: 3-way interactions } \\
\text { (change } R^{2} \text { ) }\end{array}$ & 0.00 & 0.00 & 0.00 \\
\hline $\begin{array}{l}\text { EF problems child } \times \\
\text { EF problems } \\
\text { parent } \times \text { age }\end{array}$ & -0.01 & -0.00 & 0.06 \\
\hline
\end{tabular}

$t_{P}<.10 ; * P<.05 ; * * P<.01 ; * * * P<.001$.

Abbreviations: $\mathrm{EF}$, executive functioning; $\mathrm{FR}$, father report; $\mathrm{HbA1c}$, hemoglobin A1c; MR, mother report. 

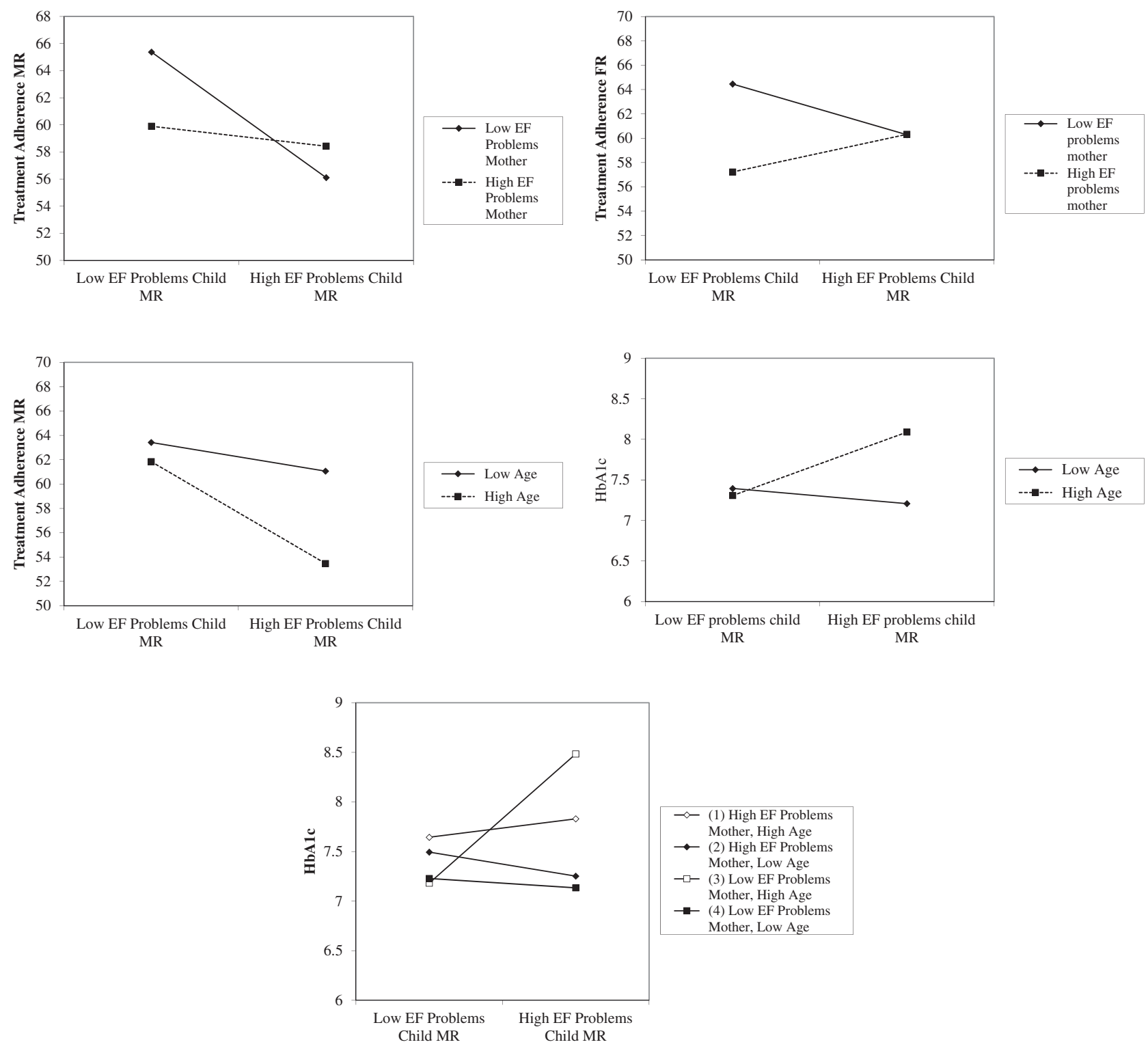

FIGURE 1 Significant interaction effects

significant in the link with $\mathrm{HbA1c}$. Further, the remaining interaction effects of child and paternal EF with age did not reach significance, nor did the 3-way interaction effect in step 4.

\section{4 | DISCUSSION}

This study extends prior work on the role of child EF in treatment adherence by examining child and parental EF in a broad developmental age range of children and adolescents with T1D and by including both mothers and fathers as informants. This allowed us to investigate interactions between child $\mathrm{EF}$, parental $\mathrm{EF}$, and age toward treatment adherence and glycemic control.

With respect to main effects, EF problems child were consistently associated with poorer treatment adherence (in mother reports) and worse $\mathrm{HbA1c}$. This confirms earlier research reporting associations between child EF and treatment adherence, ${ }^{7}$ with this study pointing to more pronounced effects for mother rather than father report of
EF. Further, contrary to expectations, parental EF was not related to treatment adherence nor $\mathrm{HbA1c}$. Such a lack of unique effects already suggests the importance of examining interactions with other variables, such as EF problems child and age, to completely understand the neuropsychological mechanisms underlying treatment adherence.

Indeed, in line with hypotheses, results pointed to a relatively consistent significant interaction effect between child and parental EF. This effect was again more prominent in mothers, suggesting that treatment adherence was highest when both mother and child showed fewer problems in EF. More specifically, results demonstrated that maternal EF problems were related to treatment adherence, especially in combination with less EF problems child. However, contrary to expectations, maternal EF did not seem to provide a protective or correcting effect on poor child EF functioning. Results therefore emphasize the importance of examining not only child but also parental EF and the difference in neuropsychological competencies between the 2 .

Further in line with our hypotheses, age was a moderator in the association between child EF and treatment adherence: more 
problems in the EF domain were associated with worse treatment adherence, especially in older children and adolescents. As stated in previous research, ${ }^{32}$ older children and adolescents increasingly take over responsibility for self-management activities from their parents, increasing the importance of their own competencies in EF. However, again, in this study these effects were only found in mother reports. Hence, age of the child seems to play a more prominent role in mothers' than in fathers' perspectives. Contrary to expectations, however, age did not function as a moderator between parental EF and treatment adherence. Therefore, age seems to be a more pronounced factor in the link between children's own EF and treatment adherence rather than in the link with parents' EF.

Finally, when examining the 3-way interactions among child EF, parental EF, and age, results partially supported our hypothesis: in older children and adolescents, more problems in the child's EF domain as reported upon by mothers were related to worse glycemic control. Interestingly, when combined with less problems in maternal $E F$, glycemic control was even worse. This may point to the fact that mothers confronted with poor glycemic control and EF problems in their child, may adjust by sharpening their own EF functions to compensate for their child's functioning, or, from a different viewpoint, to a possible negative effect of the difference between child and parental EF. However, as these results stem from a cross-sectional design, firm interpretations of directionality of effects cannot be made and should be examined in future longitudinal research.

Overall, findings suggest the importance of the main effect of EF of children and the interaction with parental EF when examining treatment adherence. Effects of child EF were more pronounced in the older age group. Further, effects were more pronounced in mother as compared to father reports. It could be that mothers, as they are often more closely involved in daily diabetes self-management tasks, ${ }^{33}$ monitor their children's (diabetes-related) behavior more closely, which might enable them to assess treatment adherence and their children's functioning more correctly. However, it should also be noted that although most effects in fathers were not statistically significant, $\beta$-values were often rather similar to those in mothers (eg, for the main effect of EF problems child on HbA1c). Therefore, the lack of effects in fathers may also be due to the lower sample size in fathers, decreasing the power to obtain significant effects.

\subsection{Limitations and suggestions for future research}

This study is characterized by some limitations. First, due to the cross-sectional design, directional interpretations should be made with caution. Future research should test longitudinal associations to allow for a more in-depth understanding of the link between child EF, parental EF, and treatment adherence in children and adolescents with T1D. Furthermore, as the development of EF depends upon cortical maturation that continues into emerging adulthood, ${ }^{34}$ future research on EF should include this age group transitioning to adult health care. Second, replication of these results with more targeted measures might be useful. Using a diabetes-specific measure such as the Diabetes Related Executive Functioning Scale $\left(D_{R E F S^{35}}\right.$ ) may be valuable. Furthermore, all of the behavioral measures were parentreported. Therefore, future research should add more objective measures of treatment adherence and EF to avoid confounding within respondents. For instance, assessing EF by means of neuropsychological testing may generate a more in-depth picture. Third, in this study children with psychiatric disorders affecting EF were excluded (ie, ADHD and autism) as these children often get special help at school and/or medication to enhance EF and broader functioning. It may be valuable for future research to include this subgroup as the co-occurrence of T1D with these psychiatric diagnoses may be additionally challenging for treatment adherence. Finally, some study characteristics such as the relatively low participation rate, the demographic characteristics, and the relatively good overall glycemic control may question the representativeness of the sample which might constitute a relatively well-functioning sample. Data for patients who declined participation are not available because of ethical considerations. However, comparisons with data from the Belgian Diabetes Registry for patients in the same age range showed that our sample showed somewhat better glycemic control (7.3\% vs $8.1 \%)$ and a somewhat shorter diabetes duration ( 4.7 vs 5.5 years). As previous studies (eg, 25) also suggest, future studies may benefit from targeting children and families in a broader ethnic and educational range as well.

\section{2 | Clinical implications}

Provided that the present findings are replicated longitudinally, translation into future interventions may be valuable. Introducing developmentally appropriate tips and tricks on core EF competencies (such as planning, shifting attention, setting priorities, etc.) into the routine educational sessions on diabetes management might be beneficial. ${ }^{21}$ Moreover, to identify children at risk for poor treatment adherence and glycemic control, assessment of EF by means of a generic (ie, $\mathrm{BRIEF}^{27}$ ) or diabetes-specific questionnaire (ie, DREFS ${ }^{36}$ ) may be useful. When there is a clinically significant indication of EF problems, targeted referral for more elaborate neuropsychological assessment is recommended. ${ }^{21,36}$ Hence, educating diabetes teams on the matter seems useful. For example, when discussing individual patientcentered decisions in terms of more complex flexible treatments or the choice between pump or pen therapy, diabetes teams may take into account a patient's level of EF. Further, as results indicate the combined positive effect of child and parental EF, clinicians should also be attentive to parental competencies in the EF domain. Therefore, ideally, parental EF is assessed as well, by means of clinical assessment in routine family anamnesis or by means of a questionnaire (eg, BRIEF- $\mathrm{A}^{28}$ ). As most interventions in the care for children and adolescents with T1D are family-based, ${ }^{37}$ integrating an additional focus on the role of neuropsychological functioning in treatment adherence in such clinical interventions might be a targeted approach. For example, psycho-education on normative developmental stages of EF in children and adolescents (ie, what to expect and not) might be valuable in helping parents to have realistic expectations and thus develop more balanced and developmentally appropriate shared responsibility with their children in self-management. This might also help parents to detect or acknowledge difficulties in their child's or own EF, opening the path for referral to even more targeted diagnosis or treatment of EF problems. 


\section{ACKNOWLEDGEMENTS}

We cordially thank all the parents who participated in the study, as well as the diabetes teams across the 7 participating hospitals for their benevolence in recruiting participants. This research was realized with the support of the Clinical Research Fund of the University Hospital Leuven granted to the first author. Partial support was granted by the VDKA (Vlaamse Diabetescentra voor Kinderen en Adolescenten).

\section{REFERENCES}

1. Hilliard ME, Harris MA, Weissberg-Benchell J. Diabetes resilience: a model of risk and protection in type 1 diabetes. Curr Diab Rep. 2012;12(6):739-748.

2. Atkinson MA, Eisenbarth GS, Michels AW. Type 1 diabetes. Lancet. 2014;383(9911):69-82

3. Cramer JA, Benedict A, Muszbek N, Keskinaslan A, Khan ZM. The significance of compliance and persistence in the treatment of diabetes, hypertension and dyslipidaemia: a review. Int J Clin Pract. 2008;62(1):76-87.

4. Osterberg L, Blaschke T. Adherence to medication. N Engl J Med. 2005;353(5):487-497.

5. Smith LB, Kugler BB, Lewin AB, Duke DC, Storch EA, Geffken GR. Executive functioning, parenting stress, and family factors as predictors of diabetes management in pediatric patients with type 1 diabetes using intensive regimens. Child Health Care. 2014;43(3):234-252.

6. La Greca A, Mackey E. Adherence to pediatric treatment regimens. In: Roberts MC, Steele RG, eds. Handbook of Pediatric Psychology. New York, NY: Routhledge University Press; 2009:130-147.

7. Duke DC, Harris MA. Executive function, adherence, and glycemic control in adolescents with type 1 diabetes: a literature review. Curr Diab Rep. 2014;14(10):1-10.

8. Rustad JK, Musselman DL, Skyler JS, et al. Decision-making in diabetes mellitus type 1. J Neuropsychiatry Clin Neurosci. 2013;25:40-50.

9. Alvarez JA, Emory E. Executive function and the frontal lobes: a meta-analytic review. Neuropsychol Rev. 2006;16(1):17-42.

10. Goldstein S, Naglieri JA, eds. Handbook of Executive Functioning. New York, NY: Springer; 2014.

11. Gilbert SJ, Burgess PW. Executive function. Curr Biol. 2008;18(3): R110-R114.

12. Blakemore SJ, Choudhury S. Development of the adolescent brain: implications for executive function and social cognition. J Child Psychol Psychiatry. 2006;47(3-4):296-312.

13. Bagner DM, Williams LB, Geffken GR, Silverstein JH, Storch EA. Type 1 diabetes in youth: the relationship between adherence and executive functioning. Child Health Care. 2007;36(2):169-179.

14. Danne T, Mortensen HB, Hougaard P, et al. Persistent differences among centers over 3 years in glycemic control and hypoglycemia in a study of 3,805 children and adolescents with type 1 diabetes from the Hvidøre Study Group. Diabetes Care. 2001;24(8):1342-1347.

15. De Luca C, Leventer R. Developmental trajectories of executive functions across the lifespan. In: Anderson V, Jacobs R, Anderson P, eds. Executive Functions and the Frontal Lobes. New York, NY: Taylor and Francis; 2014:23-56.

16. Ellis DA, Podolski CL, Frey M, Naar-King S, Wang B, Moltz K. The role of parental monitoring in adolescent health outcomes: impact on regimen adherence in youth with type 1 diabetes. J Pediatr Psychol. 2007;32:907-917.

17. Drotar D, Rohan JM. Pediatric adherence and health behavior change. In: Martin LR, DiMatteo MR, eds. The Oxford Handbook of Health Communication, Behavior Change, and Treatment Adherence. New York, NY: Oxford University Press; 2013:1-26.

18. Perantie DC, Koller JM, Weaver PM, et al. Prospectively determined impact of type 1 diabetes on brain volume during development. Diabetes. 2011;60(11):3006-3014.

19. Kirchhoff BA, Jundt DK, Doty T, Hershey T. A longitudinal investigation of cognitive function in children and adolescents with type
1 diabetes mellitus. Pediatr Diabetes. 2016. https://doi.org/10.1111/ pedi.12414.

20. Lin A, Northam EA, Rankins D, Werther GA, Cameron FJ. Neuropsychological profiles of young people with type 1 diabetes $12 \mathrm{yr}$ after disease onset. Pediatr Diabetes. 2010;11:235-243.

21. Wasserman RM, Hilliard ME, Schwartz DD, Anderson BJ. Practical strategies to enhance executive functioning and strengthen diabetes management across the lifespan. Curr Diab Rep. 2015;15(8):1-9.

22. Perez KM, Patel NJ, Lord JH, et al. Executive function in adolescents with type 1 diabetes: relationship to adherence, glycemic control, and psychosocial outcomes. J Pediatr Psychol. 2017; 42: 636-646.

23. Young MT, Lord JH, Patel NJ, Gruhn MA, Jaser SS. Good cop, bad cop: quality of parental involvement in type 1 diabetes management in youth. Curr Diab Rep. 2014;14(11):1-12.

24. Goethals ER, Oris L, Soenens B, et al. Parenting and treatment adherence in type 1 diabetes throughout adolescence and emerging adulthood. J Pediatr Psychol. 2017. https://doi.org/10.1093/jpepsy/jsx053.

25. Miller MM, Rohan JM, Delamater A, et al. Changes in executive functioning and self-management in adolescents with type 1 diabetes: a growth curve analysis. J Pediatr Psychol. 2013;38(1):18-29.

26. Craig F, Margari F, Legrottaglie AR, Palumbi R, de Giambattista $C$, Margari L. A review of executive function deficits in autism spectrum disorder and attention-deficit/hyperactivity disorder. Neuropsychiatr Dis Treat. 2016;12:1191.

27. Gioia GA, Isquith PK, Retzlaff PD, Espy KA. Confirmatory factor analysis of the Behavior Rating Inventory of Executive Function (BRIEF) in a clinical sample. Child Neuropsychol. 2002;8(4):249-257.

28. Roth, R. M., Isquith, P. K., \& Gioia, G. A. (2005). BRIEF-A. Behavior Rating Inventory of Executive Function-Adult Version Professional Manual.

29. Wysocki T, Buckloh LM, Antal H, Lochrie A, Taylor A. Validation of a self-report version of the diabetes self-management profile. Pediatr Diabetes. 2012;13(5):438-443.

30. Cohen J, Cohen P, West SG, Aiken LS. Applied Multiple Regression Correlation Analysis for the Behavioral Sciences. 3rd ed. Mahwah, NJ: Erlbaum; 2003.

31. Jaccard J, Turrisi R. Interaction Effects in Multiple Regression. 2nd ed. Thousand Oaks, CA: Sage; 2003.

32. Wiebe DJ, Chow CM, Palmer DL, et al. Developmental processes associated with longitudinal declines in parental responsibility and adherence to type 1 diabetes management across adolescence. J Pediatr Psychol. 2014;39(5):532-541.

33. Berg CA, Butner JE, Turner SL, Lansing AH, King P, Wiebe DJ. Adolescents', mothers', and fathers' reports of adherence across adolescence and their relation to $\mathrm{HbA} 1 \mathrm{c}$ and daily blood glucose. J Behav Med. 2016;39(6):1009-1019.

34. Diamond A. Normal development of prefrontal cortex from birth to young adulthood: cognitive functions, anatomy, and biochemistry. In: Stuss D, Knight R, eds. Principles of Frontal Lobe Function. New York, NY: Oxford University Press; 2002:466-503.

35. Duke DC, Raymond JK, Harris MA. The diabetes related executive functioning scale (DREFS): pilot results. Child Health Care. 2014;43(4):327-344.

36. Wasserman RM, Anderson BJ, Schwartz DD. Screening of neurocognitive and executive functioning in children, adolescents, and young adults with type 1 diabetes. Diabetes Spectr. 2016;29(4):202-207.

37. Anderson B. Family conflict and diabetes management in youth: clinical lessons from child development and diabetes research. Diabetes Spectr. 2004;17(1):22-26.

How to cite this article: Goethals ER, de Wit M, Van Broeck $\mathrm{N}$, et al. Child and parental executive functioning in type 1 diabetes: Their unique and interactive role toward treatment adherence and glycemic control. Pediatr Diabetes. 2017;1-7. https://doi.org/10.1111/pedi.12552 